\title{
Early veno-venous Extra Corporeal Membrane Oxygenation with prone positioning in the treatment of severe Acute Respiratory Distress syndrome in a pediatric patient: a case report
}

\author{
Md Nawad Azam*, Senthil Kumar S. P. \\ Department of Pediatrics and Neonatology, Columbia Asia Hospital, Whitefield, Bangalore, Karnataka, India
}

Received: 02 May 2019

Accepted: 08 May 2019

*Correspondence:

Dr. Md Nawad azam

E-mail: dr.nawed2007@gmail.com

Copyright: () the author(s), publisher and licensee Medip Academy. This is an open-access article distributed under the terms of the Creative Commons Attribution Non-Commercial License, which permits unrestricted non-commercial use, distribution, and reproduction in any medium, provided the original work is properly cited.

\begin{abstract}
Aim of the study was to discuss effectiveness and outcome of Extra Corporeal Membrane Oxygenation (ECMO) therapy with prone positioning while on ECMO in a child with severe Acute Respiratory Distress Syndrome (ARDS). Veno-venous ECMO (VV-ECMO) and prone position on ECMO are relatively newer modalities of treatment in severe ARDS in pediatric age group and to our experience very few cases has been reported in paediatric age group. A 5 year old male child presented with fever with respiratory distress. He deteriorated in next $48 \mathrm{hrs}$ leading to severe hypoxemia with ARDS following which he was put on conventional ventilation. In view of worsening critical lung parameters, veno-venous ECMO was initiated. Child had persistent hypoxemia inspite on high positive end expiaratory pressure (PEEP), adequate flow and adequate Post oxygenator $\mathrm{PO}_{2}$. Recirculation and other possible causes were excluded. Prone positioning (PP) was done for $8 \mathrm{hrs}$ a day with chest physiotherapy while on ECMO. Patient showed considerable improvement in next few days and ECMO was weaned on day 7 and child was discharged on day 16 with good lung recovery. Although studies on VV-ECMO with PP has proven effectiveness in severe ARDS in adult population, overall studies in paediatric ARDS are very limited. Prone positioning on ECMO may decrease duration of ECMO support. Prone positioning on VV ECMO is safe and can be considered in Persistent hypoxemia with poor respiratory compliance.
\end{abstract}

Keywords: ARDS, Conventional ventilation, ECMO, Hypoxemia, Positive end expiaratory pressure, Prone positioning

\section{INTRODUCTION}

Acute respiratory distress syndrome (ARDS) in pediatric age group still remains one of the challenging clinical condition for intensivist. According to AmericanEuropean Consensus Conference (EACC) ARDS was defined as: the acute onset of respiratory failure, bilateral infiltrates on chest radiograph, hypoxemia as defined by a $\mathrm{PaO} 2 / \mathrm{FiO} 2$ ratio $\leq 200 \mathrm{mmHg}$, and no evidence of left atrial hypertension or a pulmonary capillary pressure $<18$ $\mathrm{mmHg}$ (if measured) to rule out cardiogenic edema. ${ }^{1} \mathrm{But}$ recently the new Berlin definition of ARDS has been published which suggested severity-oriented respiratory treatment by introducing three levels of severity (mild, moderate, and severe) according to $\mathrm{PaO}_{2} / \mathrm{FiO}_{2}{ }^{2}$

Due to high rates of mortality many lung protective strategies and adjuncts has been opted like low Tidal volume $(\mathrm{Vt})$, high PEEP, Prone positioning, Nitric oxide (NO),Surfactant, HFOV,ECMO with variable success rates. ${ }^{3}$ Recent studies have shed some light on potential mechanisms of prone positioning by demonstrating that ARDS patients have inhomogeneous distribution of alveolar collapse and that patients in the prone position 
appear to have more recruitment of atelectatic dorsal lung regions. ${ }^{4}$ In case of failure of conventional treatment, ECMO is currently the last resort. VV-ECMO is considered to support children with severe ARDS where the cause of the respiratory failure is believed to be reversible. ${ }^{5}$ Combining one or more of these lung protective strategies has shown marked reduction in refractory hypoxia of severe ARDS. ${ }^{6}$

\section{CASE REPORT}

5 year old male child with past history of seizure disorder with moderate global developmental delay presented with fever, mild tachypnea and saturation of $88 \%$ in RA. Initial chest X-ray showed clear lung field (Figure 1A). He was found to have Total leucocyte count of 4000/mm3 with $91 \%$ neutrophil with high CRP (increased 10 times of cut off value). Nasal swab for H1N1 PCR was sent and found to be negative for influenza. He was initially started on $\mathrm{O} 2$ at $4 \mathrm{lt} / \mathrm{min}$, IV Ceftriaxone, Inj. Azithromycin and nebulization. Child showed progressive increase in work of breathing with high grade fever and desaturation on $\mathrm{O} 2$ at $10 \mathrm{lts} / \mathrm{min}$.

On 4th day of admission he developed excessive frothing and bleeding from mouth with decreased sensorium and impending respiratory failure. Repeat chest X-ray showed bilateral fluffy opacities (Figure 1B) and ABG showed respiratory acidosis with hypercapnea $(60 \mathrm{mmHg})$ and hypoxia. He was immediately put on conventional ventilation PRVC mode with FIO2-100\%, PEEP-10, TV230ml, Rate-30/min. Antibiotics were upgraded to Meropenem and Linezolid. Child was also put on Vasopressors in view of low Invasive arterial BP and poor perfusion. Child continue to deteriorate on conventional ventilation requiring very high PIP (45-46), high MAP (>20), FOI2-100\% to deliver tidal volume at $6 \mathrm{ml} / \mathrm{kg}$. PaO2/FIO2 was 65 , Oxygen index was well above 30 for 6 hrs associated with refractory shock.

In view of alarming deterioration of child's condition decision was taken to put the patient on early rescue Veno-venous ECMO. Child was sedated with neuromuscular relaxation. ECMO cannulation done with Size17 Fr for femoral drainage and 20Fr SVC input under trans-esophageal ultrasound guidance (Figure $2 \mathrm{~A}$ and $\mathrm{B}$ ). Position was confirmed with chest $\mathrm{x}$-ray (Figure $1 \mathrm{C}$ ). ECMO setting were as followed- flow gradually increased to $2.3 \mathrm{lts} / \mathrm{min}$, sweep flow-4 lts/min, Trans membrane pressure-45. Ventilator setting was gradually decreased to TV-100ml, FIO2- $40 \%$, rate-16/min. PEEP was continued at 10 to prevent atelectasis. Child had persistent low $\mathrm{PaO} 2$ inspite of adequate flow, sweep flow with post membrane $\mathrm{PaO} 2>400 \mathrm{~mm} \mathrm{Hg}$. Re-circulation was excluded. In hope of better respiratory toileting and ventilation of atelectatic dorsal lung field, child was put on prone position for 8 hrs every day for 3 days on ECMO. Child showed marked improvement in ventilation on day 6 of VV-ECMO with Pao2/FIO2-
172.8, Oxygen index-5.7 with marked clearing of lung field on chest X-ray (Figure $1 \mathrm{D})$.

Patient was put on total parenteral nutrition and hematocrit was optimized with blood transfusions. Child was gradually weaned of mechanical ventilation and put on High flow nasal cannula (HFNC). He was gradually weaned of oxygen and discharged in good health and near $100 \%$ lung recovery.
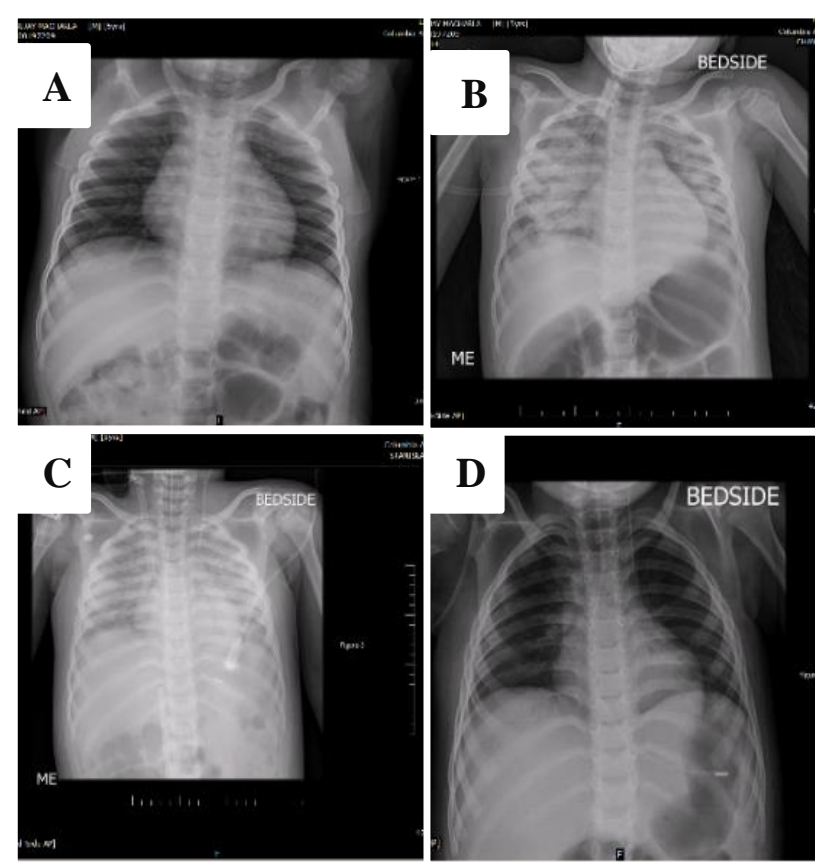

Figure 1: Chest X-ray changes of the patient during the course of hospitalization. (A): Apparently clear lung field at the time of admission, (B): Full blown

ARDS feature in the same patient by Day 4, (C): ECMO catheter position in Chest X-ray, (D): Cleared lung field at the time of discharge.

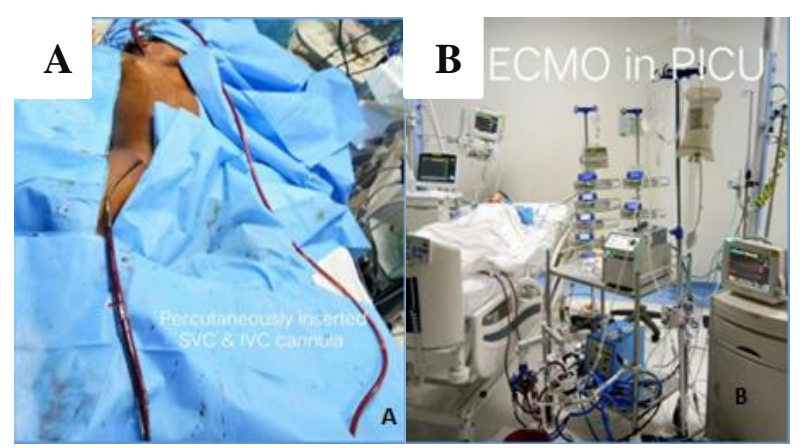

Figure 2 (A): Percutaneous inserted SVC and IVC cannula, 2 (B): ECMO set up in PICU.

\section{DISCUSSION}

Recent advances in critical care has brought down mortality related to ARDS from $80-90 \%$ once to $30-50 \%$ in most series depending on underlying disease process. ${ }^{7}$ 
Many lung protective strategies with adjuncts has been studied in adults but similar series of studies are lacking in pediatric ARDS which is forcing pediatric critical care clinician to rely and project outcome of adult cases in pediatric age group.

Although effectiveness of early ECMO in neonatal age group is well established, Paediatric ECMO is less frequent and the clinical indications are more heterogeneous. This may be due to the lack of a large RCT looking at the efficacy of ECMO in advanced respiratory failure. Appropriate sizes of cannula for drainage and inflow and their proper positioning is must for reducing recirculation and drainage issues in $\mathrm{VV}$ ECMO ${ }^{8}$ Hollow fiber membrane oxygenator and better understanding of the ECLS physiology has increased the effectiveness of veno-venous ECMO in pediatric respiratory failure. ${ }^{9}$ Early initiation VV-ECMO with oxygen index $>25$ for more than 6 hours with high PIP requirement may have more favorable neurological and functional outcome. ${ }^{10}$

Recent adult studies have demonstrated an improvement in the $\mathrm{PaO} 2 / \mathrm{FIO} 2$ ratio by $20 \mathrm{~mm} \mathrm{Hg}$ in ARDS patients who were prone-positioned as shown by L'Her et al in their study of 51 patients with ARDS due to variety of underlying illnesses (pneumonia, sepsis, lung contusion, multiple trauma). ${ }^{11}$ Although exact mechanism of improvement due to prone positioning is unknown it may be attributed to multiple reasons like better recruitment of atelectatic dorsal lung region, optimizing mobilization and drainage of secretion. Combining prone positioning with VV-ECMO has shown considerable improvement in cases with high platue pressure, severe hypoxemia $(\mathrm{PaO} 2 / \mathrm{FIO} 2<70)$, failure to wean off ECMO. ${ }^{12}$ In our case sudden and critical deterioration of patient's condition prompted us to early VV-ECMO and prone positioning not only expedite the clinical improvement but also helps attaining the full functional recovery of the patient. Logistics \& the financial constraints still remain a major setback in the initiation of ECMO programme in many centers in developing countries like India.

\section{CONCLUSION}

Although studies on VV-ECMO with PP has proven effectiveness in severe ARDS in adult population, overall studies in paediatric ARDS are very limited. Prone positioning on ECMO may decrease duration of ECMO support. Prone positioning on VV ECMO is safe and can be considered in Persistent hypoxemia with poor respiratory compliance.

\section{ACKNOWLEDGEMENTS}

Authors would like to thank Dr. Naveenchandra from Department of Cardiology, Dr. Arun Reddy, Cardiovascular surgeon, Dr. Subhasmita, Cardiac anaesthetist, CAHW for valuable support in the management of the child.
Funding: No funding sources

Conflict of interest: None declared

Ethical approval: Not required

\section{REFERENCES}

1. Bernard GR, Artigas A, Brigham KL, Carlet J, Falke K, Hudson L, et al. The American-European Consensus Conference on ARDS. Definitions, mechanisms, relevant outcomes, and clinical trial coordination. Am J Respir Crit Care Med. 1994;149(3 Pt 1):818-824.

2. ARDS Definition Task Force, Ranieri VM, Rubenfeld GD, Thompson BT, Ferguson ND, Caldwell E, et al. Acute respiratory distress syndrome: the Berlin Definition. JAMA. 2012 Jun 20;307(23):2526-33.

3. Anderson MR. Update on Pediatric Acute Respiratory Distress Syndrome. Resp Care. March 2003;48(3):261-278.

4. Gattinoni L, Bombino M, Pelosi P, Lissoni A, Pesenti A, Fumagalli R, et al. Lung structure and function in different stages of severe adult respiratory distress syndrome. JAMA. 1994 Jun 8;271(22):1772-9.

5. The Pediatric Acute Lung Injury Consensus Conference Group (2015). Pediatric Acute Respiratory Distress Syndrome: Consensus Recommendations from the Pediatric Acute Lung Injury Consensus Conference. Pediatr Crit Care Med. 2015;16(5),428-439.

6. D Ng, W Klein, R Tran, D Riddle-Branske, PJ Luna, HB Nguyen. Combination therapy with highfrequency oscillatory ventilation, neuromuscular blockade, inhaled nitric oxide and prone position in acute respiratory distress syndrome with refractory hypoxaemia. Anaesth intensive care. 2012;40(5):898-9.

7. Davis SL, Furman DP, Costarino AT Jr. Adult respiratory distress syndrome in children: associated disease, clinical course, and predictors of death. J Pediatr. 1993 Jul;123(1):35-45.

8. Conrad SA, Grier LR, Scott LK, Green R, Jordan M. Percutaneous cannulation for extracorporeal membrane oxygenation by intensivists: a retrospective single-institution case series. Crit Care Med. 2015 May;43(5):1010-5.

9. Iwahashi H, Yuri K, Nosé Y. Development of the oxygenator: past, present, and future. J Artif Organs. 2004;7(3):111-20.

10. Rivera RA, Butt W, Shann F. Predictors of mortality in children with respiratory failure: possible indications for ECMO. Anaesth Intensive Care. 1990 Aug; 18(3):385-9.

11. L'Her E, Renault A, Oger E, Robaux MA, Boles JM. A prospective survey of early 12-h prone positioning effects in patients with the acute respiratory distress syndrome. Intensive Care Med. 2002 May;28(5):570-5. 
12. Guervilly C, Hraiech S, Gariboldi V, Xeridat F, Dizier S, Toesca R, et al. Prone positioning during veno-venous extracorporeal membrane oxygenation for severe acute respiratory distress syndrome in adults. Minerva Anestesiol. 2014 Mar;80(3):307-13.
Cite this article as: Azam MN, Kumar SSP. Early veno-venous Extra Corporeal Membrane

Oxygenation with prone positioning in the treatment of severe Acute Respiratory Distress syndrome in a pediatric patient: a case report. Int J Contemp Pediatr 2019;6:1743-6. 